\title{
REVISIÓN
}

REVIEW

$\overline{1}$ Licenciado en Fonoaudiología. Carrera de Fonoaudiología. Departamento de Procesos Terapéuticos, Facultad Ciencias de la

Salud. Universidad Católica de Temuco, Chile.

${ }^{2}$ Fonoaudiólogo, Magíster en Neuropsicología. Carrera de Fonoaudiología, Departamento de

Especialidades Médicas. Universidad de La Frontera. Temuco, Chile.
Correspondencia a: Camilo Morales.

camilo.morales@uct.cl

Telf. y celular: $+452-25533875$

Palabras clave: Deglución; Trastornos de Deglución; Calidad de Vida

Keywords: Deglutition; Deglutition Disorders; Quality of Life

Procedencia y arbitraje: no comisionado, sometido a arbitraje externo.

Recibido para publicación:

21 de Abril de 2018

Aceptado para publicación:

14 de Julio de 2018

Citar como:

Rev Cient Cienc Med

2018; 21 (1):78-83

\section{CALIDAD DE VIDA RELACIONADA A LA DE- GLUCIÓN CONCEPTOS Y APLICABILIDAD EN SALUD}

Camilo Morales ${ }^{1}$, Exequiel Guevara ${ }^{2}$.

\section{RESUMEN}

La disfagia o trastorno de la deglución es una complicación clínica prevalente con impacto en la salud y calidad de vida de las personas, entendiéndose esta última como la percepción del individuo sobre su salud en variadas dimensiones, cuya medición permite captar impacto de la enfermedad o progresos terapéuticos. Su utilidad clínica en la valoración de la disfagia implica además captar la perspectiva de la persona en relacion a sus propias complicaciones. El objetivo del presente estudio es establecer una revisión teórica del concepto calidad de vida y sus variaciones, además de ofrecer y describir instrumentos genéricos y específicos de utilidad para la medición del constructo, especificando en los trastornos deglutorios. Para tal fin, se llevó a cabo una revisión de literatura en bases de datos para la identificación y descripción de los principales instrumentos utilizados. Se discute la utilidad clínica, en investigación y elementos a considerar para aplicar dichos instrumentos en diferentes contextos.

\section{ABSTRACT}

Dysphagia or swallowing disorder is a prevalent clinical complication, with an impact on the health and people's quality of life. The later being understood as the individual's perception of their health in various dimensions, whose measurement allows to describe the impact of the disease or therapeutic progress. Its clinical utility in the assessment of dysphagia also involves capturing the person's perspective of their own complications. The objective of this study is to establish a theoretical review of the concept of quality of life and its variations, in addition to offering and describing generic and specific instruments of utility for the measurement of the construct, specifying in swallowing disorders. For this purpose, a literature review was carried out in databases to identify and describe the main instruments used. The clinical or research utility and elements to consider in order to apply those instruments in different contexts are discussed.

\section{INTRODUCCIÓN}

a disfagia orofaríngea (DO), o dificultad para

deglutir, comprende un conjunto de alteraciones estructurales, funcionales, con compromiso sensorial, motriz o conductual que impacta negativamente ya sea en el procesamiento o paso del alimento de la boca hacia el estómago'. Su prevalencia varía, dependiendo de la población objetivo. Los adultos mayores (AM) están en mayor riesgo debido a su dispar eficacia y seguridad en la función deglutoria, causada de manera característica por una propulsión lingual débil y una respuesta del reflejo deglutorio más retrasada atribuible a la edad. Turley et $\mathrm{al}^{2}$, estimaron la prevalencia de disfagia en $13,7 \%$ para esta población. Martino et al $^{3}$ encontraron una prevalencia de aproximadamente $55 \%$ en personas hospitalizadas con accidente cerebrovascular (ACV). En consecuencia, es una condición frecuente, particularmente en los AM y pacientes con enfermedades neurológicas"; no obstante, su origen responde a múltiples factores, observándose también en sujetos con condiciones congénitas, 
procesos neoplásicos, traumatismos craneales, entre otros ${ }^{5}$.

Esta complicación tiene consecuencias severas para la salud de los usuarios, la cual puede llevar a deshidratación, malnutrición o neumonía por aspiración ${ }^{4}$. Se evidencian además efectos a un nivel psicológico, en donde los riesgos de sofocación, tos refleja severa o vómitos pueden causar un incremento de la ansiedad o menor autoestima6.

Desde una perspectiva social, los momentos de alimentación pueden ser muy estresantes, como por ejemplo, asistir a un restaurant o celebraciones de diversa índole pueden dejar de ser placenteros debido a la presencia de alguna dificultad para deglutir 6 .

El proceso de intervención de estos trastornos, y sus consecuencias, consiste principalmente en diversos ejercicios terapéuticos, cambios posturales, y conductuales; estos últimos dicen relación con la modificación de volúmenes y consistencias del alimento a ingerir, los cuales son indicados para compensar las manifestaciones de esta alteración, además de asegurar y volver más agradable la deglución. Sin embargo, en reiteradas ocasiones las mediciones de los progresos terapéuticos se objetivan sin tomar en cuenta la percepción del usuario respecto a este tipo de manejo, generando como consecuencia que puedan ser ampliamente rechazados, pues cabe la posibilidad de que éstos no impacten directamente sobre la Calidad de Vida (CV) de las personas. Esta conceptualización es a menudo confusa y requiere de una adecuada delimitación, específicamente para los procesos de evaluación y tratamiento, donde en muchas ocasiones no se considera la perspectiva del usuario. Resulta necesario, por tanto, contar con instrumentos que permitan medir con exactitud y precisión este constructo.

El objetivo del siguiente estudio consiste en ofrecer al lector una visión sobre el concepto de Calidad de Vida y sus aproximaciones en salud, sus posibles aplicaciones clínicas o con fines meramente investigativos, enfatizando en la medición de este constructo en usuarios con Disfagia. Asimismo, se presenta una revisión con algunos de los instrumentos utilizados actualmente de manera genérica y específica para realizar este tipo de valoraciones.

\section{METOdOLOGIA DE BÚSQUEDA DE ARTículos}

Se realizó una búsqueda en la base de datos Pubmed, utilizando los siguientes términos y códigos booleanos: ((Dysphagia OR Deglutition OR "Deglutition disorders"[Mesh] OR swallowing OR "swallowing disorders" OR "swallowing problems") AND ("Quality of Life"[Mesh] OR "HRQoL" OR "health-related quality of life") AND ("Validation Studies as Topic"[Mesh] OR validity OR "validation" OR reliability OR reproducibility OR "Reliability of Results" OR "Reproducibility of Results"[Mesh]). La búsqueda generó 231 resultados iniciales, seleccionando como filtros metodológicos "revisiones sistemáticas de literatura" y "estudios de validación", con la finalidad de identificar aquellos instrumentos que estén actualmente en utilización. Se seleccionaron finalmente 15 de ellos basados en el título, abstract, relevancia del tema y antigüedad (menor a 10 años). Por otra parte, se efectúa una segunda revisión de tipo clásico según lo propuesto Montero y León ${ }^{3}$, en donde se utilizan artículos extraídos de otros motores de búsqueda y bibliografía pertinente para complementar lo encontrado en la búsqueda principal.

\section{DESARROLLO}

\section{CALIDAD DE VIDA}

Se entiende Calidad de Vida (CV) como la percepción del individuo sobre su salud, considerando aspectos sociales, psicológicos y funcionales ${ }^{7}$. Como consecuencia de esta conceptualización, existen bastantes investigaciones tendientes a intentar medir este constructo, sin que haya demasiada claridad respecto a la utilización del término. En relación a esto, la Organización Mundial de la Salud (OMS) ${ }^{8}$ define CV como: "la percepción individual de la propia posición en la vida dentro del contexto del sistema cultural y de valores en que se vive y en relación con sus objetivos, esperanzas, normas y preocupaciones". Por ello, la calidad de vida se establece de acuerdo con un sistema de valores, estándares o perspectivas que varían entre las propias personas, grupos o lugares ${ }^{9}$.

Dentro de las opciones que existen para poder medir CV, buena parte se basa en diversos modelos, lo cual tributa a la falta de consenso acerca de la definición específica de lo que implica este constructo. Por ejemplo, el modelo de necesidades, que concibe a la CV como la capacidad de los usuarios de satisfacer sus necesidades humanas; dentro de esta, menciona aspectos como la identidad, autoestima, amor, seguridad, creatividad, sueño, entre otras. Otro ejemplo sería el modelo de reintegración a la vida normal; definiéndose como la habilidad de la persona de hacer cosas que debe o desea hacer, sin que necesariamente se asocie con la ausencia de enfermedad ${ }^{10}$. Claramente, para poder realizar la medición de éstos, se requiere la confección de instrumentos que evalúen precisamente estos dominios e intenten relacionarlos, para asi poder realizar las estimaciones pertinentes. Sin embargo, su medición en muchos casos no resulta específica ni útil para el clínico, el cual se interesa por reconocer aspectos relacionados con resultados específicos en salud y su impacto en la CV.

\section{CALIDAD DE VIDA RELACIONADA A LA SALUD}

Debido a la confusión conceptual reportada por la literatura, existen múltiples definiciones para el concepto de Calidad de Vida Relacionada con la Salud (CVRS), por el cual se solapa con el de CV descrito anteriormente. No es objetivo del estudio profundizar sobre éstas, sino, en primera instancia, establecer una diferenciación terminológica que permita definir con claridad lo que se pretende estudiar.

De acuerdo a lo mencionado por Urzúa ${ }^{11}$, se define CVRS 
como "El nivel de bienestar derivado de la evaluación que la persona realiza de diversos dominios de su vida, considerando el impacto que en éstos tiene su estado de salud". Es así que, mientras el concepto de CV enfatiza en aspectos psicosociales (cuantitativos o cualitativos) del bienestar social; la CVRS se enfoca en la evaluación de parte del individuo, sus limitaciones en áreas biológicas, psicológicas y sociales a raíz de una enfermedad, condición o accidente ${ }^{12}$.

Pese a las diferencias conceptuales, la gran mayoría de los autores sugieren que la CV se diferencia de CVRS, debido a que este último se utiliza en ámbitos de salud en su interés por evaluar la calidad de los cambios como resultado de intervenciones médicas, pues debe limitar su abordaje a la experiencia que el paciente tiene de su enfermedad, porque caracterizan la experiencia del paciente resultado de los cuidados médicos o para establecer el impacto de la enfermedad en la vida diaria, es decir, es una medición de salud desde la perspectiva de los pacientes ${ }^{11}$.

Se ha operacionalizado, por tanto, la CVRS en diferentes modelos, de los cuales han resultado algunos instrumentos de medición. Dentro de sus dimensiones, se incluyen las "Percepciones Generales de Salud" (PGS), definida como la evaluación que el individuo hace de los efectos que tiene su salud sobre otros aspectos de la vida y el "Estado Funcional de Salud" (EFS) o habilidad del individuo de poder realizar normalmente actividades diarias, requeridas para satisfacer necesidades básicas, cumplir roles y mantener la salud y el bienestar ${ }^{13}$. En otras palabras, la limitación de la funcionalidad por la influencia de una enfermedad específica, incluyéndose el funcionamiento físico, psicológico y social.

Se considera al EFS como una medida de utilidad clínica tanto para profesionales médicos como no médicos, respecto a la consideración de la severidad de los signos clínicos desde la perspectiva del usuario, pues valora la habilidad de desempeñar tareas en dominios físico, social, roles y funcionamiento psicológico, entre otros ${ }^{14}$. Hay autores que consideran al EFS dentro del constructo de CVRS $^{14}$; otros mencionan que constituye un concepto diferente ${ }^{4}$, precisamente porque este último es más complejo desde el punto de vista de los aspectos que mide (y de acuerdo al modelo utilizado) y el primero se centra principalmente en las temáticas relacionadas a la funcionalidad y desempeño en actividades de la vida diaria. Por esto, no resulta extraño identificar elementos de CVRS en instrumentos de medición de EFS y viceversa ${ }^{14}$.

\section{MEDICIÓN DE CVRS Y EFS}

Si bien existen múltiples formas de evaluar la severidad del trastorno desde la estructura y funciones del individuo, a menudo no se considera la perspectiva del paciente sobre su propio desempeño, vivencias y el impacto que su enfermedad tiene sobre su persona. Por tanto, las medidas de CVRS y EFS contribuyen a obtener esta información para el clínico, con miras a la generación de planes terapéuticos pertinentes. Generalmente se constituyen a cuestionarios, los cuales son auto-administrados, fáciles de utilizar y no requieren mucho tiempo o esfuerzo por parte del clínico o el paciente ${ }^{15}$. Junto con ello, se utilizan para evaluar el curso de una enfermedad o los efectos de los tratamientos, por lo cual pueden ser una importante herramienta tanto en la identificación de problemas y la facilitación de decisiones terapéuticas, como en el monitoreo de cambios clínicos ${ }^{15}$. El valor de reportar los resultados que derivan del paciente usando estos cuestionarios específicos ha sido apreciado por muchos años ${ }^{16}$. Asimismo, a menudo se utilizan como unidad de medida primaria en ensayos clínicos, particularmente cuando no existe medida de beneficio directo para capturar el bienestar del paciente ${ }^{17}$, es decir, como una medida de efectividad terapéutica.

Es así, que la selección y utilización de los cuestionarios que serán incluidos dentro del proceso de evaluación, dependerán tanto de las necesidades de los profesionales de la salud como de los pacientes ${ }^{18}$. Existen cuestionarios genéricos (que proveen una descripción general del estado de salud percibido por los pacientes) y específicos (que facilitan el pronóstico o permiten medir la progresión terapéutica). Dentro de estos últimos, existe una amplia variedad que permiten valorar CVRS o EFS en individuos con disfagia o en riesgo de padecerla ${ }^{4}$, sin embargo, no todos estos son factibles de aplicar en contextos clínicos, dada su complejidad, especificidad o simplemente por que no se encuentran validados en la realidad local, lo que conduce a errores en medición. A continuación, se ofrece al lector una recopilación y descripción de instrumentos genéricos o específicos para la medición de estos constructos:

\section{MEDIDAS GENÉRICAS}

EQ-5D: Cuestionario genérico y estandarizado, auto-administrable, fácil de responder y con poca carga cognitiva. Su uso se ha generalizado en todo el mundo, encontrándose disponible en más de 170 idiomas, incluido el español en varias versiones. Se compone de tres partes: la primera permite al encuestado definir el estado de salud según el sistema de clasificación multi-atributo EQ-5D, compuesto por 5 dimensiones (movilidad, cuidado personal, actividades cotidianas, dolor/malestar y ansiedad/depresión), y en cada una de ellas existen 3 niveles de gravedad $(1,2$ o 3 ); la segunda parte es una escala visual analógica graduada de 0 (peor estado de salud imaginable) a 100 (mejor estado de salud imaginable), que permite al individuo valorar el estado de salud y obtener un índice de utilidad de su CVRS dividiendo el valor señalado en la escala entre 100; finalmente, la tercera parte recoge otros datos anónimos que permiten hacer una caracterización demográfica del grupo estudiado ${ }^{19}$. 
SF-36: Escala genérica que proporciona un perfil del estado de salud, aplicable tanto a pacientes como a la población general. Ha resultado útil para: evaluar la CVRS en la población general y en subgrupos específicos, comparar la carga de muy diversas enfermedades, detectar los beneficios en la salud producidos por un amplio rango de tratamientos diferentes y valorar el estado de salud de pacientes individuales. El Cuestionario de Salud SF-36 está compuesto por 36 preguntas (ítems) que valoran los estados tanto positivos como negativos de la salud, distribuidas en 8 escalas: Función Física (10 ítems), Rol Físico (4 ítems), Dolor Corporal (2 ítems), Salud General (5 ítems), Vitalidad (4 ítems), Función Social (2 ítems), Rol Emocional (3 ítems) y Salud Mental (5 ítems). Adicionalmente, el SF-36 incluye un ítem de transición que pregunta sobre el cambio en el estado de salud general respecto al año anterior. Se encuentra disponible para personas mayores de 14 años e idealmente debería ser auto-administrado. Las escalas del SF-36 están ordenadas de forma que a mayor puntuación mejor es el estado de salud. De esta manera, un puntaje mayor o menor a 50 puntos (de 100 en total) en cada una de ellas indicaría mejor o peor estado de salud ${ }^{20}$.

\section{MEDIDAS ESPECÍFICAS PARA LA DEGLUCIÓN}

SWAL-QOL: El SWAL-QOL es considerado como el "Gold Standard" para determinar CVRS en personas con disfagia orofaríngea. Está construido sobre la base de un modelo genérico de calidad de vida, abordando algunas dimensiones de este constructo. Está compuesta por 44 ítems agrupados en 11 sub-escalas: 10 dominios basados en calidad de vida y una escala sintomatológica. Estos dominios incluyen: (1) Carga de enfermedad, (2) Selección de alimentos, (3) Duración de la alimentación, (4) Deseo de comer, (5) Miedo, (6) Sueño/Descanso, (7) Fatiga, (8) Comunicación, (9) Salud mental y (10) Funcionamiento Social ${ }^{21}$. Es el instrumento más utilizado en investigaciones para determinar si la disfagia orofaríngea impacta la calidad de vida de las personas. Con esta finalidad, explora este constructo dentro de varias etiologías, tales como cáncer orofaríngeo, enfermedad tiroidea, esclerosis lateral amiotrófica, entre otras ${ }^{22}$.

MD Anderson Dysphagia Inventory (MDADI): Constituida por 20 ítems, está diseñada para medir CVRS. Además de una evaluación global correspondiente a una sola pregunta, comprende tres sub-escalas: la emocional (8 ítems), la funcional (5 ítems) y la física (6 ítems). Se puntúa cada ítem de manera que los puntajes mayores son indicadores de mejor funcionamiento deglutorio. Posee evidencia de uso y buenas propiedades psicométricas principalmente en la valoración de los trastornos deglutorios de personas con cáncer de cabeza y cuello ${ }^{23}$.

Deglutition Handicap Index (DHI): Busca medir la CVRS. Fue creado como herramienta clínica para valorar trastornos deglutorios de cualquier etiología, basado en el modelo del Voice Handicap Index. El objetivo fue crear una herra- mienta práctica para evaluar el impacto de la deglución en el usuario siendo, sin embargo, más breve que SWAL-QOL y menos específico que el MDADI. Mide 3 dimensiones: (1) emocional, (2) funcional y (3) física. Contiene 30 ítems en un formato de respuesta de escala de 5 puntos, en términos de frecuencia. Sus puntajes varían de 0 a 120; mientras más puntuación, mayor discapacidad ${ }^{24}$.

Swallowing Outcome after Laryngectomy (SOAL): Evalúa EFS. Consiste en una sola escala con 17 ítems (de 0 a 2 puntos máximo), diseñada específicamente para valorar la percepción de función deglutoria posterior a laringectomía total. El puntaje mínimo es 0 y el máximo es de 34 . Mientras mayor sea el puntaje, mayor será la alteración funcional en la deglución ${ }^{25}$.

Sydney Swallowing Questionnaire (SSQ): Es un cuestionario de una escala, consistente en 17 preguntas de tipo escala visual análoga (de 0 a 100) específicamente diseñadas para evaluar las disfuncionalidades de la fase oral y faríngea de la deglución. Por ende, mide EFS. El puntaje mínimo es de 0 y el máximo de 1700 . Mientras mayor el puntaje final, mayor compromiso funcional de la deglución. Los autores consideran a este instrumento valioso para valorar y tratar la disfagia en usuarios con cáncer orofaríngeo ${ }^{26}$.

Dysphagia in Multiple Sclerosis questionnaire (DYMUS): Cuestionario diseñado para evaluar la funcionalidad deglutoria de usuarios con esclerosis múltiple. Mide EFS. Consiste en 10 ítems con respuestas dicotomizadas, conformadas por dos escalas: la escala de "disfagia para sólidos" (7 ítems) y la escala de "disfagia para líquidos" (3 ítems). La puntuación final varía entre 0 a 10; los puntajes más altos indican una función deglutoria más severa ${ }^{27}$.

Eating Assessment Tool (EAT-10): Cuestionario auto-administrado, que se enfoca principalmente en EFS para una valoración sobre la percepción de la disfagia. Instrumento conformado por 10 ítems, cuyas respuestas se puntúan utilizando una escala de 5 puntos, resultando así una puntuación final que varía desde los 0 a los 40 . Se considera una puntuación de 3 o mayor sugerente de un problema en el proceso deglutorio. Este cuestionario se diseñó para ser utilizado en un amplio rango de usuarios con disfagia, de diferentes etiologías, para la valoración de la severidad de los síntomas, calidad de vida y eficacia terapéutica ${ }^{28}$.

\section{DISCUSIÓN}

Las valoraciones de CVRS o EFS deben ser consideradas como parte de la evaluación de la Disfagia Orofaríngea. Estas mediciones pretenden capturar la percepción única y personal de la salud del individuo, tomando en cuenta los factores sociales, funcionales y psicológicos, permitiendo a los profesionales de la salud, tanto médicos como no médicos, tomar en cuenta aspectos de la severidad de los síntomas del trastorno deglutorio desde la perspectiva del paciente, práctica que al parecer no siempre ejecutada. Se 
utilizan diferentes técnicas para obtener esta información relevante acerca de estos aspectos, siendo la más utilizada por medio de los cuestionarios auto-administrados, debido a que el método de recolección de datos es más estructurado y estandarizado en comparación con una entrevista. Al utilizar estos instrumentos en el ámbito clínico, se establecen valores o categorías que permiten en muchos casos determinar decisiones acerca del diagnóstico o futuro tratamiento ${ }^{29}$.

La confección de herramientas para la medición de constructos subjetivos como el EFS o la CVRS constituye un proceso complejo, el cual debe desarrollarse de manera cuidadosa para garantizar sus propiedades de validez (relacionado con la interpretación y el significado de la puntuación de un instrumento o, en otras palabras, las conclusiones que se pueden realizar con el puntaje obtenido por una persona), confiabilidad (mediciones realizadas de manera que sean reproducibles: repetidas por diferentes observadores o en distintas ocasiones) y utilidad (si resulta práctico su uso en entornos clínicos o no), es decir, que cumpla su función tanto en la valoración del concepto que se pretende medir, como para el entorno y las condiciones actuales de la población de estudio ${ }^{30}$. De este modo, si bien estos instrumentos se encuentran traducidos y validados para diferentes países ${ }^{22,31}$ resulta oportuno para el clínico verificar si existen versiones aptas para su aplicación en la población del país perteneciente, dadas las variaciones del constructo propias de características lingüísticas, culturales o clínicas.

La utilización de instrumentos de medición no validados en una población específica puede constituir un sesgo, ya que los constructos o sus dimensiones pueden variar de acuerdo a diferentes características. La falta de instrumentos validados en diferentes poblaciones, a su vez, limita la generalización de los resultados de la efectividad de un tratamiento determinado a nivel clínico, además de la sistematización y reporte a la comunidad científica. En la medida que la diversidad aumenta en estudios de efectividad de tratamientos, se deben desarrollar y aplicar instrumentos que den cuenta acerca de la evidencia de validez de resultados en grupos poblacionales diversos ${ }^{32}$, considerando la ubicación geográfica, características culturales, variables sociodemográficas, entre otras. De este modo, constituye un desafío el adaptar o generar nuevos instrumentos pertinentes a la población a ser evaluada cuando sea necesario.

\section{CONCLUSIONES}

La CVRS y el EFS son conceptos que todo clínico debiese considerar a la hora de efectuar una evaluación o un plan de intervención, ya sea en un usuario que presente disfagia u otra condición, con la finalidad de complementar estos procesos, pues consideran tanto las percepciones sintomatológicas como también necesidades de los usuarios, familiares y cuidadores

Asimismo, con el presente estudio se espera lograr sensibilizar al personal que se desempeña en el ambiente sanitario, logrando así que puedan complementar su práctica laboral con estos importantes conceptos, pues surgen como una alternativa para el monitoreo y reporte de los cambios clínicos pre y post intervención y, por tanto son medidas relevantes en este contexto.

Finalmente, se insta a investigadores y clínicos a indagar más en este tópico, desarrollando estudios de validación de instrumentos como los presentados, que consideren tanto las características particulares de cada población, como la variabilidad de los constructos relativos a calidad de vida, pues dependen del contexto en el cual se apliquen. De esta manera, se garantizaría la validez y confiabilidad en la medición de estas variables latentes.

\section{REFERENCIAS}

1.Logemann JA. Introduction: Definitions and Basic Principles of Evaluation and Treatment of Swallowing Disorders. In: Evaluation and Treatment of Swallowing Disorders. 2nd ed. Austin: PRO-ED; 1998. p. 1-3.

2.Turley $\mathrm{R}$, Cohen $\mathrm{S}$. Impact of voice and swallowing problems in the elderly. Otolaryngol - Head Neck Surg [Internet]. 2009;140(1):33-6. Disponible en: http://dx.doi.org/10.1016/j. otohns.2008.10.010

3.Martino R, Foley N, Bhogal S, Diamant N, Speechley M, Teasell $R$. Dysphagia after stroke: Incidence, diagnosis, and pulmonary complications. Stroke. 2005;36(12):2756-63.

4.Speyer R, Kertscher B, Cordier R. Functional Health Status in Oropharyngeal Dysphagia. J Gastroenterol Hepatol Res [Internet]. 2014;3(5):1043-8. Disponible en: http://www.ghrnet.org/index.php/ joghr/article/view/692

5.Turner MA. Dysphagia Revisited: Common and Unusual
Causes 1. 2015;(2):105-22.

6.Ekberg O, Hamdy S, Woisard V, Wuttge-Hannig A, Ortega P. Social and psychological burden of dysphagia: Its impact on diagnosis and treatment. Dysphagia. 2002;17(2):139-46.

7.Gutiérrez-Achury AM, Ruales-Suárez K, Giraldo-Cadavid LF, Rengifo-Varona ML. Escalas de calidad de vida y valoración de los síntomas en disfagia. R Med. 2015;23(November 2016):52-7.

8.World Health Organization. Division of Health Promotion, Education and C. Health Promotion Glossary [Internet]. World Health Organization. Geneva; 1998. Disponible en: http://www.who. int/healthpromotion/about/HPR Glossary 1998.pdf

9.Velarde-jurado E, Avila-figueroa C. Evaluación de la calidad de vida. Salud Publica Mex [Internet]. 2002;44(4):349-61. Disponible en: http://www.scielo.org.mx/pdf/spm/v44n4/14023.pdf

10.Fayers PM, Machin D. Quality of Life. The assessment, analysis and interpretation of patient-reported outcomes. 2nd ed. Vol. 14. Chichester: Wiley \& Sons Ltd.; 2007. 7 p. 
11.Urzúa M A. Calidad de vida relacionada con la salud: Elementos conceptuales. Rev Med Chil. 2010;138(3):358-65.

12. Alpi SV, Quiceno JM. Calidad de vida relacionada con la salud y enfermedad crónica: estudios colombianos. Psychol AV DISCIP [Internet]. 2012;6(1):123-36. Disponible en: http://www.redalyc.org/ articulo.oa? $\mathrm{id}=297225770004$

13.Feasley JC. Health Related Quality of Life. In: Health Outcomes for Older People: Questions for the Coming Decade. Washington D.C: National Academy of Sciences; 1996. p. 17-9.

14.Morrison RS, Meier DE. Geriatric Palliative Care. New York: Oxford University Press; 2003. 23-25 p.

15.Deshpande PR, Rajan S, Sudeepthi BL, Abdul Nazir CP. Patientreported outcomes: A new era in clinical research. Perspect Clin Res [Internet]. 2011;2(4):137-44. Disponible en: http://www. pubmedcentral.nih.gov/articlerender.fcgi?artid=3227331\&tool $=$ pmcentrez\&rendertype $=$ abstract

16.Feasley JC. Health Outcomes Research for Older People. In: Health Outcomes for Older People: Questions for the Coming Decade. Washington D.C: National Academy of Sciences; 1996. p. 9-10.

17.Feeny DH, Eckstrom E, Whitlock EP, Perdue LA. A Primer for Systematic Reviewers on the Measurement of Functional Status and Health-Related Quality of Life in Older Adults. AHRQ [Internet]. 2013; Disponible en: http://www.ncbi.nlm.nih.gov/ pubmed/24199257

18.Mark DB. Assessing quality-of-life outcomes in cardiovascular clinical research. Nat Rev Cardiol [Internet]. 2016; Disponible en: http://dx.doi.org/10.1038/nrcardio.2016.10

19.Pradas Velasco R, Villar FA, Puy Martínez-Zárate M. Utilización del cuestionario European Quality of Life-5 Dimensions (EQ-5D) para valorar la variación de la calidad de vida relacionada con la salud debida a la gripe. Gac Sanit. 2009;23(2):104-8.

20.Vilagut G, Ferrer M, Rajmil L, Rebollo P, Permanyer-Miralda G, Quintana JM, et al. El Cuestionario de Salud SF-36 español: una década de experiencia y nuevos desarrollos. Gac Sanit [Internet]. 2005;19(2):135-50. Disponible en: http://linkinghub.elsevier.com/ retrieve/pii/S0213911105713418

21.McHorney CA, Robbins J, Lomax K, Rosenbek JC, Chignell K, Kramer AE, et al. The SWAL-QOL and SWAL-CARE outcomes tool for oropharyngeal dysphagia in adults: III. Documentation of reliability and validity. Dysphagia. 2002;17(2):97-114.

22. Woisard V, Sordes F. Health Related Quality of Life and Oropharyngeal Dysphagia. J Gastroenterol Hepatol Res [Internet].
2014;3(10):1292-300. Disponible en: http://www.healthypeople. gov/2020/about/qolwbabout.aspx

23.Guedes R, Chen A, Vartanian J, Carrara-De Angelis E. Validation and application of the md anderson dysphagia inventory in head and neck cancer patients. Dysphagia [Internet]. 2011;26(4):458. Disponible en: http://www.embase.com/search/ results?subaction $=\quad$ viewrecord \& from $=$ export\&id $=$ L 70663834 nhttp://dx.doi.org/10.1007/s00455-011-9345-1 \nhttp://sfx.library. uu.nl/utrecht?sid=EMBASE\&issn $=\quad$ 0179051X\&id=doi:10.1007/ s00455-011-9345-1\&atitle $=$ Valiation + and + application

24.Crestani S, Moerman M, Woisard V. The "deglutition handicap index" a self-adminitrated dysphagia-specific quality of life questionnaire: Sensibility to change. Rev Laryngol Otol Rhinol. 2011;132(1):3-7.

25.Govender R, Lee MT, Drinnan M, Davies T, Twinn C, Hilari $\mathrm{K}$. Psychometric evaluation of the Swallowing Outcomes after Laryngectomy (SOAL) patient-reported outcome measure. Head Neck. 2016;38:E1639-45.

26.Dwivedi RC, St. Rose S, Roe JWG, Khan AS, Pepper C, Nutting $\mathrm{CM}$, et al. Validation of the sydney swallow questionnaire (SSQ) in a cohort of head and neck cancer patients. Vol. 27, Dysphagia. 2012. p. 285.

27.Alali D, Ballard K, Vucic S, Bogaardt $H$. Dysphagia in Multiple Sclerosis: Evaluation and Validation of the DYMUS Questionnaire. Dysphagia. 2017;1-9.

28.Belafsky PC, Mouadeb DA, Rees CJ, Pryor JC, Postma GN, Allen $\mathrm{J}$, et al. Validity and Reliability of the Eating Assessment Tool (EAT-10). Ann Otol Rhinol Laryngol. 2008;117(12):919-24.

29.Alarcón AM, Muñoz S. Medición en salud : Algunas consideraciones metodológicas. Rev Med Chile. 2008;136:125-30. 30.Keszei AP, Novak M, Streiner DL. Introduction to health measurement scales. JPsychosom Res [Internet]. 2010;68(4):319-23. Disponible en: http://dx.doi.org/10.1016/j.jpsychores.2010.01.006

31.Timmerman AA, Speyer R, Heijnen BJ, Klijn-Zwijnenberg IR. Psychometric characteristics of health-related quality-oflife questionnaires in oropharyngeal dysphagia. Dysphagia. 2014;29(2):183-98.

32.Patrick DL, Chiang YP. Measurement of health outcomes in treatment effectiveness evaluations: conceptual and methodological challenges. Med Care. 2000;38(9):II14-I25. 